\title{
Lithospheric thinning due to hydration and melting at oceanic transform plate boundaries
}

\section{Zhikai Wang}

University of Paris

Satish Singh ( $\nabla$ singh@ipgp.fr)

Institut De Physique Du Globe De Paris https://orcid.org/0000-0002-4594-801X

Cecile Prigent

University of Paris

Emma Gregory

University of Paris

Milena Marjanovic

Institut De Physique Du Globe De Paris

\section{Article}

Keywords: transform faults, tectonics, transform plate boundaries

Posted Date: March 31st, 2021

DOI: https://doi.org/10.21203/rs.3.rs-255780/v1

License: (c) (1) This work is licensed under a Creative Commons Attribution 4.0 International License.

Read Full License

Version of Record: A version of this preprint was published at Nature Geoscience on August 25th, 2022. See the published version at https://doi.org/10.1038/s41561-022-01003-3. 


\section{Abstract}

Transform plate boundaries, one of the key elements of plate tectonics, accommodate lateral motions and produce large earthquakes, but their nature at depth remains enigmatic. Using ultra-long offset seismic data, here we report the presence of a low-velocity anomaly extending down to $\sim 60 \mathrm{~km}$ depth beneath the Romanche transform fault in the equatorial Atlantic Ocean. Our result indicates the presence of deep penetration of water leading to extensive serpentinization down to $16 \mathrm{~km}$, followed by a shear mylonite zone down to $32 \mathrm{~km}$ over a low-temperature water induced-melting zone, elevating the lithosphere-asthenosphere boundary and hence thinning the lithosphere significantly beneath the transform fault. The presence of a thinned lithosphere and the melt underneath could lead to volcanism, migration and mixing of the water-induced melt with the high-temperature melt beneath the ridge axis, and small-scale convections beneath transform boundaries. Hence, a thinned lithosphere will have a major impact on the dynamics of ridge-transform system, and will influence the evolution of fracture zones and oceanic lithosphere.

\section{Main Text}

Transform faults (TFs) were discovered by Tuzo Wilson in $1965^{1}$ as a new class of plate boundaries that accommodate relative lateral motions of two adjacent lithospheric plates. TFs host a large number of earthquakes ${ }^{2}$, which can sometimes be devastating ${ }^{3}$. TFs are oceanic or continental, depending on the type of lithosphere they crosscut. Oceanic TFs often offset two segments of mid-ocean ridges ${ }^{1}$. They comprise almost one-fifth of the total length of active global plate boundaries ${ }^{4}$, with a total length of $\sim 48,000 \mathrm{~km}$, but release sixteen times more seismic energy than the $\sim 67,000 \mathrm{~km}$-long spreading ridges ${ }^{5}$. Away from active transform boundaries, the oceanic TFs are preserved as fracture zones (FZs), which extend over entire ocean basins and form some of the most prominent and continuous features on the ocean floor ${ }^{2}$. These prominent FZs sink into the mantle at subduction zones, and fundamentally affect the seismic and magmatic dynamics of subduction zones ${ }^{6}$. The crustal structures of a few oceanic TFs have been studied recently through geophysical surveys ${ }^{7,8}$, but the properties and nature of the deep lithosphere and the lithosphere-asthenosphere boundary (LAB) at oceanic TFs remain unfathomed. Here, we present the velocity structure of the lithosphere beneath the Romanche TF down to $60 \mathrm{~km}$ depth below the sea surface (bsf).

\section{Study area}

Our study area lies in the equatorial Atlantic Ocean across the Romanche TF (Fig. 1), which offsets the slow-spreading Mid-Atlantic Ridge (MAR) by $\sim 880 \mathrm{~km}$ and has hosted several large earthquakes in the last 40 years $^{9}$. As the full slip rate ${ }^{10}$ at the MAR is $\sim 32 \mathrm{~mm} / \mathrm{yr}$, the Romanche TF is among the largest and fastest active strike-slip plate boundaries on the Earth, comparable to its continental counterparts: the Dead Sea Fault ( $1100 \mathrm{~km}$ long, slip rate of $\sim 7.2 \mathrm{~mm} / \mathrm{yr})$, the North Anatolian Fault ( $1500 \mathrm{~km}$ long, slip rate of $\sim 24 \mathrm{~mm} / \mathrm{yr}$ ) and the San Andreas Fault ( 1200 km long, slip rate of $\sim 42 \mathrm{~mm} / \mathrm{yr})^{11}$. The great 
equatorial Atlantic TFs of St. Paul TF, Romanche TF and Chain TF from north to south, with a total offset of $\sim 1800 \mathrm{~km}$, were responsible for the break-up of Africa with South America $\sim 110 \mathrm{Myr}$ ago $^{12}$. Therefore, investigating the structures beneath these features is important not only for the understanding of the strike-slip plate boundaries, but also for the continental break-ups.

We acquired an $850 \mathrm{~km}$-long wide-angle seismic refraction profile using ocean-bottom seismographs (OBSs) (see Methods), crossing the Romanche TF (Fig. 1). The age contrast across the Romanche TF along the profile is $\sim 32 \mathrm{Myr}$, with $8 \mathrm{Myr}$-old lithosphere to the south and $40 \mathrm{Myr}$-old lithosphere to the north ${ }^{13}$. In this region, the Romanche TF is characterized by a $\sim 20 \mathrm{~km}$ wide transform valley and a hanging basin on the northern flank. Previous studies ${ }^{14}$ have suggested that the vicinity of the eastern ridge-transform intersection (RTI) of the Romanche TF is cold and crust-free. However, recent seismic studies suggested that the crust is 5.5-6.5 km thick beneath the Romanche TF ${ }^{8}$ and the LAB lies at $~ 72$ $\mathrm{km}$ depth beneath the 40 Myr-old lithosphere north of the transform ${ }^{15}$, arguing for the presence of a normal lithosphere in this region.

\section{Mantle velocity structure beneath the Romanche TF}

The OBS data quality is excellent; the mantle refraction (Pn) arrivals are observed to $250 \mathrm{~km}$ offsets for most OBSs, and six OBSs record Pn arrivals up to $700 \mathrm{~km}$ offsets (Fig. 2 and Supplementary Fig. 2).

The nearly flat Pn arrivals on the recorded pressure data (Fig. 2 and Supplementary Figs. 2,3) reduced by $8.0 \mathrm{~km} / \mathrm{s}$ velocity suggest that the mean P-wave velocity in the ridge-parallel direction for the uppermost mantle along the seismic profile is consistent with that $(7.9-8.1 \mathrm{~km} / \mathrm{s})$ observed in other regions of the Mid-Atlantic Ocean ${ }^{16}$. The travel time delays and weak amplitudes observed on several OBSs (pointed out by red arrows in Supplementary Fig. 3) for the rays entering the Romanche TF imply the possible presence of a low-velocity structure in the mantle beneath the Romanche TF.

To investigate the velocity structure of the lithosphere, we first invert the manually picked arrival times of the crustal refractions and the reflections from the Mohorovičic discontinuity (Moho) to characterize the crustal velocity and the depth of the Moho along the profile (Fig. 3a). Our results are consistent with crustal structures obtained from other independent seismic studies ${ }^{8,17}$. Then the mantle velocity is estimated by inverting the manually picked $\mathrm{Pn}$ arrival times using a 2-D ray-based travel time tomography method (see Methods).

The tomographic result (Fig. 3a) shows that the lithosphere beneath the Romanche TF is characterized by a deep-rooted low-velocity anomaly (LVA), extending down to $\sim 60 \mathrm{~km}$ depth bsf. The LVA is well defined above $35 \mathrm{~km}$ depth, and lies beneath the Romanche transform valley, but widens below this depth, possibly due to the limited ray paths (Supplementary Fig. 5) or the smoothing effect of tomography (Supplementary Figs. 6-8).

Below the transform valley, the mantle velocity is $\sim 7.30 \mathrm{~km} / \mathrm{s}$ just below the Moho, which corresponds to a $\sim 8 \%$ reduction in velocity relative to the surrounding mantle velocity $(\sim 7.85-8.0 \mathrm{~km} / \mathrm{s}$; Fig. 3a). The 
velocity reduction of the LVA decreases with depth to $\sim 1 \%$ at $60 \mathrm{~km}$ depth. The tomographic vertical mantle velocity gradient within the LVA decreases rapidly from $\sim 50 \mathrm{~m} / \mathrm{s} / \mathrm{km}$ just below the Moho to $\sim 18$ $\mathrm{m} / \mathrm{s} / \mathrm{km}$ at $16 \mathrm{~km}$ depth, then slowly to $\sim 11 \mathrm{~m} / \mathrm{s} / \mathrm{km}$ at $32 \mathrm{~km}$ depth and remains nearly constant underneath with some slight undulations, revealing three distinct zones in depth range (Fig. 3b).

The smearing studies using simulated models (see Methods; Supplementary Fig. 6) indicate that the LVA down to $60 \mathrm{~km}$ depth is not produced by the smearing effect of tomography and is therefore real. The resolution studies (see Methods; Supplementary Figs. 7,8) demonstrate that the real decrease of mantle velocity within the LVA zone beneath the Romanche TF is likely to be much larger than that estimated by the tomographic method. This is because the ray-based tomography method used here can only provide a smooth velocity structure, underestimating the velocity decrease significantly in the mantle beneath the Romanche TF. Furthermore, synthetic inversion tests demonstrate that the trends in the vertical mantle velocity gradient (Fig. 3b) cannot be accounted for by smoothing effects of tomography (Supplementary Figs. $7 \mathrm{~h}, 8 \mathrm{~h}$ ), and therefore they are a real representation of the velocity trend in the mantle beneath the TF. To quantify the real mantle velocity structure beneath the Romanche TF that would lead to the tomographically inverted LVA, we performed 60 inversion tests using simulated models (see Methods). The synthetic models are designed by introducing LVA with varying percentage in velocity reductions at different depths and varying width into a modified velocity model (Supplementary Fig. 9). Rays traveling in these simulated models are computed using the same source-receiver geometry as that of the Pn picks, and the modelled travel times are inverted employing the same tomography method used for the real OBS data. The resulting 60 inverted models (Supplementary Fig. 11) are then compared with the tomographic velocity obtained using the real OBS dataset (see Methods; Supplementary Table 1). Based on these comparisons, we found that the mantle velocity within the LVA increases from $6.89 \pm 0.12 \mathrm{~km} / \mathrm{s}$ just below the Moho to $7.75 \pm 0.06 \mathrm{~km} / \mathrm{s}$ at $60 \mathrm{~km}$ depth, with the width of the LVA varying from $34.1 \pm 3.9 \mathrm{~km}$ to $35.6 \pm 5.7 \mathrm{~km}$, respectively (see Methods; Fig. $3 \mathrm{c}$ ). These estimates suggest that the real decrease in the LVA just below the Moho could be $\sim 12.4 \%$ and at $60 \mathrm{~km}$ depth it could be $\sim 4.5 \%$. Based on these results and the change in the vertical mantle velocity gradient (Figs. 3b,c), the lithospheric mantle beneath the Romanche TF can be divided into three zones: Zone 1 from the Moho down to $16 \mathrm{~km}$ depth, Zone 2 from $16 \mathrm{~km}$ to $32 \mathrm{~km}$ depth and Zone 3 below $32 \mathrm{~km}$ down to $60 \mathrm{~km}$ depth.

\section{Mantle hydration}

Combined with the petrological constraints available from oceanic TFs, we interpret the LVA at the Romanche TF as being caused by hydration and/or deformation of the upper mantle down to $60 \mathrm{~km}$ depth, where hydration leads to mantle alterations (Zones 1 and 2 ) and hydrous mantle melting (Zone 3 ).

Brittle deformation induced by earthquakes on oceanic TFs could create and sustain high-permeability pathways for seawater-derived fluids to penetrate deep into the lithosphere ${ }^{2}$. Resulting fluid-rock interactions and metamorphic reactions affect the compositional, structural and mechanical properties of TFs, and consequently, seismic velocities. Brittle deformation at oceanic TFs has been suggested to occur down to the $\sim 600^{\circ} \mathrm{C}$ isotherm 9,18 . However, recent petrological studies on peridotite mylonites 
dredged from some oceanic TFs suggest that seawater-derived fluids infiltrate down to at least 850$875^{\circ} \mathrm{C}^{19,20}$, implying brittle deformation and mantle hydration at higher temperatures.

\section{Serpentinized zone}

Petrological observations from natural samples collected on oceanic TFs show that fluid interaction with mantle rocks leads to metamorphic reactions whose nature is temperature dependent ${ }^{19,20}$. The fluid-rock interactions typically result in serpentinization of mantle peridotites below $\sim 500-550^{\circ} \mathrm{C}^{19}, 20$, triggering a significant decrease in seismic velocity ${ }^{21}$. At the Romanche TF, the plate cooling mode ${ }^{22}$ predicts a mantle temperature of $450^{\circ} \mathrm{C}$ at $16 \mathrm{~km}$ depth (Supplementary Fig. 13). As this temperature is consistent with the upper stability limit of serpentine ${ }^{19-21}$, we interpret Zone 1 to represent the serpentinized fault zone, delimited by the rapid change in vertical mantle velocity gradient at $16 \mathrm{~km}$ depth (Fig. $3 \mathrm{~b}$ ). In this case, $\sim 55 \pm 7 \%$ of mantle serpentinization would be required to account for the $12.4 \%$ decrease in velocity just below the Moho, decreasing to $25 \pm 5 \%$ at $16 \mathrm{~km}$ depth (see Methods; Supplementary Fig. 14). This depth is also coincident with the maximum focal depth of large earthquakes that have occurred in this region ${ }^{9}$, supporting our interpretation.

\section{Mylonite zone}

Above $\sim 600^{\circ} \mathrm{C}$, peridotites on oceanic TFs transition from a brittle to a semi-brittle behavior ${ }^{19}, 20$. Episodes of brittle deformation in the semi-brittle zone create transient-permeability pathways that drive penetration of seawater-derived fluids and hydration reactions ${ }^{19,20}$, leading to the formation of hydrated and fine-grained mylonitic peridotites. The observed change in velocity gradient at $32 \mathrm{~km}$ depth (Fig. $3 \mathrm{~b}$ ) coincides with the $920^{\circ} \mathrm{C}$ isotherm (Supplementary Fig. 13). Though it is slightly higher than the estimated maximum temperature $\left(850-875^{\circ} \mathrm{C}\right)$ for the production of hydrated mylonites on oceanic TFs ${ }^{19}$, 20 , we interpret this depth as the base of the mylonite zone (Zone 2).

The hydration of peridotites could lead to the crystallization of hydrous minerals, such as chlorite and amphibole ${ }^{19,20}$ that exhibit lower seismic velocities than peridotites ${ }^{23}$. Assuming that the mantle velocity anomaly in Zone 2 is mainly due to this hydration processes, the estimated fraction of hydrous minerals would decrease from $28 \pm 6 \%$ at $16 \mathrm{~km}$ depth to $10 \pm 7 \%$ at $32 \mathrm{~km}$ depth (see Methods; Supplementary Fig. 15). Using the estimated fraction of hydrous minerals within natural samples of peridotite mylonite ${ }^{19}$, our calculations suggest that $70 \pm 15 \%$ of the mantle at $16 \mathrm{~km}$ depth and more than $24 \%$ of the mantle at 32 $\mathrm{km}$ depth would be mylonitic at the Romanche TF (Supplementary Fig. 15). Although the change in velocity gradient at $16 \mathrm{~km}$ depth is rather sharp (Fig. 3b), there might be a transition zone where serpentine, chlorite and amphibole co-exist. A microseismic study from this region highlights a band of microseismicity between $18 \mathrm{~km}$ and $34 \mathrm{~km}$ depths $\mathrm{bsf}^{24}$, which is consistent with our interpretation of a zone made of hydrated mylonites at these depths.

\section{Mantle Melting}


The LVA beneath $32 \mathrm{~km}$ depth at the Romanche TF, with a large and nearly constant vertical velocity gradient, cannot simply be explained by mantle anisotropy, temperature and variations in composition. Mantle anisotropy could decrease the seismic velocity in the orthogonal direction of shear deformation, but it could not have produced a large and positive vertical mantle velocity gradient in this direction ${ }^{25}$. An excess temperature (Supplementary Fig. 16b) due to thermal diffusion cannot account for the lowvelocity structure, because the required temperature to reduce the velocity exceeds the solidus of mantle peridotite (see Methods). The presence of metasomatic peridotites ${ }^{14}$ could lower the mantle velocity, however, the plagioclase formation indicates that metasomatic reactions occur shallower than $\sim 32 \mathrm{~km}$ depth bsf ${ }^{14}$.

The LVA below $32 \mathrm{~km}$ depth could be explained by the presence of a small amount of melt in the mantle. Assuming the mantle beneath the Romanche TF starts melting at $32 \mathrm{~km}$ depth and using the effective medium theory ${ }^{26}$, the estimated mantle velocities (Fig. 3c) would require $1.75 \pm 0.30 \mathrm{wt} \%$ and $1.11 \pm 0.16$ wt\% of melt at $32 \mathrm{~km}$ and $60 \mathrm{~km}$ depths, respectively, in the form of thin films with an aspect ratio of 50 (see Methods; Supplementary Fig. 17). Such thin films of melt could be produced by the localized shear deformations within the transform zone, and has been proposed to explain the low seismic velocity in the LAB beneath the equatorial Atlantic Ocean ${ }^{15}$.

\section{Role of water}

The temperature required to melt a dry mantle below $32 \mathrm{~km} \mathrm{depth}^{27}$ (Supplementary Fig. 18) is much higher than the predicted temperature of the Romanche TF (Supplementary Fig. 13) using the plate cooling model ${ }^{22}$. The presence of volatiles, such as water and/or carbon dioxide, in the mantle is critical to produce hydrous melt at low temperatures ${ }^{27,28}$. At the Romanche TF, the predicted temperature of $920^{\circ} \mathrm{C}$ at $32 \mathrm{~km}$ depth (Supplementary Fig. 13 ) is very close to the minimum temperature $\left(\sim 922^{\circ} \mathrm{C}\right.$ (ref. 27); Supplementary Fig. 18) for melting a water-saturated mantle at this depth. Therefore, the water could be the main volatile that drives hydrous mantle melting between $32 \mathrm{~km}$ and $60 \mathrm{~km}$ depth beneath the Romanche TF. To trigger hydrous mantle melting at these depths and the corresponding temperaturepressure conditions, the required water fraction would be $0.32 \mathrm{wt} \%$ at $\sim 32 \mathrm{~km}$ depth decreasing to 0.02 wt\% at 60 km depth (see Methods; Supplementary Fig. 18).

The hydrous mantle melting brings out the question of the source of water. One possibility is that water within the shallow lithosphere penetrates down to $32 \mathrm{~km}$ depth, facilitated by the seismicity-related fracturing within the brittle and semi-brittle lithosphere. But the amount of downward percolating water should be small, because the extreme thermal gradient at the Romanche TF would counteract the downward water diffusion. Another possible source of water is the sub-lithospheric water-rich melt channel at the base of the oceanic lithosphere observed in the equatorial Atlantic region ${ }^{15}$. The top boundary of this channel lies at $\sim 72 \mathrm{~km}$ depth north of the Romanche TF and corresponds to the $1260^{\circ} \mathrm{C}$ isotherm for the 40 Myr-old lithosphere ${ }^{15}$. If this boundary south of the Romanche TF also corresponds to $1260^{\circ} \mathrm{C}$ isotherm, it would lie at $\sim 36 \mathrm{~km}$ depth for the $8 \mathrm{Myr}$-old lithosphere (Supplementary Fig. 13). 
Thermal diffusion from the hotter/younger lithosphere to the colder/older lithosphere could also shallow the LAB. Mehouachi and Singh ${ }^{15}$ noted that the top of the melt channel was shifted by $15-20 \mathrm{~km}$ towards the colder lithosphere beneath the St. Paul FZ, supporting the idea of shallowing of the LAB. In addition, the Romanche transform valley is $\sim 20 \mathrm{~km}$ wide in our study area, and is $<10 \mathrm{~km}$ wide at the eastern RTI, indicating some extensions in this region ${ }^{29}$. Trans-tension along TF would further uplift the LAB to a shallower depth, modifying the geotherm and consequently facilitating the hydrous mantle melting. At 32 $\mathrm{km}$ depth, our estimated water content derived from melt is close to the upper limit of the water content estimated using hydrous minerals (Supplementary Fig. 19), implying that the two sources of hydrous melt could exist simultaneously.

\section{Concluding remarks}

The lithospheric mantle beneath the Romanche TF can be divided into three distinct zones (Fig. 4): (1) A serpentinized zone from the Moho down to $16 \mathrm{~km}$ depth bsf where the serpentization decreases from $55 \pm 7 \%$ to $25 \pm 5 \%$, representing the brittle mantle. (2) A hydrous and mylonitic zone with the fraction of hydrous minerals decreasing from $28 \pm 6 \%$ at $16 \mathrm{~km}$ depth to $10 \pm 7 \%$ at $32 \mathrm{~km}$ depth bsf. This zone is consistent with the depth range where microearthquakes occur ${ }^{24}$, forming a semi-brittle mantle zone. (3) A water-induced melt zone underneath, where the presence of water triggers hydrous mantle melting at a relatively low temperature, connected with the high-temperature sub-lithospheric melt channel ${ }^{15}$ below.

Our simulated tomographic study indicates a sharp boundary between the Romanche transform zone and the normal oceanic lithosphere on either side, but there could be a transition zone between the deep hydration zone beneath the TF and the normal oceanic lithosphere. Although we have used the uncertainties in the estimated widths as transition zones (Fig. 4), the real transition might be much more complex, especially for the water-induced melt zone. Secondly, we have used a ray-based tomographic method, which assumes infinite frequency, possibly leading to slightly higher uncertainties in the velocity estimation. Thirdly, we have used the $1260^{\circ} \mathrm{C}$ isotherm to represent the top of the $L A B$ for the younger lithosphere south of the TF, but the real temperature might be lower ${ }^{30}$, and hence the depth of the LAB might be shallower. In any case, the top of the LAB would be at least 10-15 km shallower than the surrounding lithosphere, and the lithosphere would be much thinner beneath the oceanic TFs. If the $920^{\circ} \mathrm{C}$ isotherm constrains the depth of the lithosphere, then the lithosphere would be thin along the whole transform segment.

The presence of thin lithosphere would have a significant effect on the geodynamics of the ridgetransform system. It might account for some recent observations of thick magmatic crust at oceanic $\mathrm{FZ}^{17}$ and TF ${ }^{8}$. The occurrence of volcanisms ${ }^{31}$ along oceanic TFs might be caused by the presence of a thin lithosphere. The absence of wide-spread volcanisms along TFs could be explained by efficient cooling from hydrothermal circulations along faults ${ }^{32}$.

Some recent numerical modelling studies indicate shallow and flat isotherms beneath oceanic TFs ${ }^{32}$. The presence of a thinned lithosphere and the melt underneath could lead to migration and mixing of the 
water-induced melt with the upwelling melt beneath the ridge axis. The petrology of basalts recovered along the MAR segment close to the eastern RTI of the Romanche TF shows increased water content in the melt approaching the Romanche $\mathrm{TF}^{33}$, supporting the idea of the melt migration and mixing. The presence of melt beneath a dome-shaped LAB (Fig. 4) could also initiate a small-scale convection beneath TFs. On the other hand, leaky transforms ${ }^{34}$ might be manifestations of melt movement along thinned lithosphere. A thin lithosphere beneath TFs would also influence the evolution of fracture zones, and subsequently the evolution of oceanic lithosphere.

The high degree hydration down to $>30 \mathrm{~km}$ depth suggests that a large amount of water, and possibly other volatiles, is stored within oceanic TFs. These volatiles will get released through dehydration process at subduction zones, affecting earthquake generation processes, mantle melting and arc volcanism ${ }^{6}$. There have been suggestions that oceanic FZs can act as sites for giant earthquake nucleation ${ }^{35}$ and may also help to create segmentation along subduction zones ${ }^{6}$. Moreover, the reactivated FZs on oceanic plates have the ability to trigger destructive earthquakes ${ }^{36}$, and may even lead to the development of nascent plate boundaries ${ }^{37}$. Deep rooted hydration and deformation along TF would facilitate these processes.

LVAs observed on major active continental TFs generally penetrate down to mid-lower crust ${ }^{38,39}$. At the central and eastern North Anatolian Fault, LVA is reported to merge into the asthenosphere ${ }^{40}$, but it extends much shallower in the Marmara Sea ${ }^{41}$. This implies that continental TFs exhibit a complicated structure. Although the rheology and thermal structure of the lithosphere might be different, the mylonitization of the lithosphere due to localized shear deformation and partial melting at depth could be common features along oceanic and continental TFs.

\section{Methods}

Data acquisition. The ILAB-SPARC experiment was conducted in the equatorial Atlantic Ocean on board the French R/V 'Pourquoi Pas?' in August-October 2018. The ILAB-SPARC N-S profile was shot in a southsoutheast-north-northwest direction. Fifty four-component ocean bottom seismographs (OBSs) were deployed on the seafloor at $\sim 14.2 \mathrm{~km}$ interval. The shot line was extended by $50 \mathrm{~km}$ south of OBS50 and by $100 \mathrm{~km}$ north of OBS01, giving the N-S profile a total length of $\sim 850 \mathrm{~km}$. The seismic source was composed of two sub-arrays with eight guns on each array, producing a total volume of 4990 cubic inches. The airgun arrays were towed at $10 \mathrm{~m}$ depth below the sea level and were fired every $300 \mathrm{~m}$. The vessel speed varied from 3.2 to 4.5 knots during acquisition. The maximum recording time for each shot is $130 \mathrm{~s}$ and the sampling rate is $4 \mathrm{~ms}$. The pressure component of the acquired seismic data is used in this study.

Initial velocity model for ray-based tomography. Ray-based tomography requires an initial estimate of the velocity structure for the target region. The velocity structure of our study area is composed of four layers: water layer, sedimentary layer (where present), igneous crust and upper mantle. The thickness of the 
water column is obtained from the high-resolution bathymetry data acquired during the ILAB-SPARC expedition. We use a constant water velocity of $1.5 \mathrm{~km} / \mathrm{s}$ in tomography. Even though the water velocity exhibits some small horizontal and vertical variations, numerical tests demonstrate that using a constant water velocity of $1.5 \mathrm{~km} / \mathrm{s}$ has minor influence on the modelled ray paths and travel times.

The thickness of sediments is estimated using the two-way travel times (TWTTs) of the basement events, assuming the average velocity of sediment is $1.86 \mathrm{~km} / \mathrm{s}$ (ref. 8, 17). The TWTTs of the basement events are manually picked on the migrated image of the seismic reflection data acquired along the profile.

The crustal velocity and the depth of the Moho in the study region are constrained by the travel times of crustal refractions $(\mathrm{Pg})$ and Moho reflections $(\mathrm{PmP})$, using a method similar to Gregory et al. ${ }^{8}$ and Marjanovic et al. ${ }^{17}$. Then the crustal velocity and the depth of the Moho are held fixed whilst performing tomography using mantle refractions $(\mathrm{Pn})$.

The initial mantle velocity is a 1D linear gradient model hung from the Moho. The mantle velocity increases from $7.8 \mathrm{~km} / \mathrm{s}$ with a velocity gradient of $14.0 \mathrm{~m} / \mathrm{s} / \mathrm{km}$ for the top $20 \mathrm{~km}$ (ref. 25), then gently increases with a velocity gradient of $3.5 \mathrm{~m} / \mathrm{s} / \mathrm{km}$. The model is $866 \mathrm{~km}$ wide and $90 \mathrm{~km}$ deep, and is discretized by $300 \mathrm{~m}$ grid spacing horizontally and $60 \mathrm{~m}$ grid spacing vertically. Numerical tests demonstrate that further reducing the grid sizes had negligible effect on calculated travel times.

Ray-based travel time tomography of mantle refractions. In this section, we introduce the tomography using the Pn arrivals, and readers can refer to Gregory et al. ${ }^{8}$ and Marjanovic et al. ${ }^{17}$ for the tomography of the Pg and PmP arrivals. The seismic data are filtered by a 4-5-12.5-15 Hz bandpass filter before picking the Pn arrivals. The picking uncertainty assigned to the Pn picks is $45 \mathrm{~ms}, 65 \mathrm{~ms}$ or $100 \mathrm{~ms}$, depending on the signal-to-noise ratio of the data. During ray-based tomography, the ray paths from sources to receivers and the corresponding travel times are calculated using the shortest path method ${ }^{42}$. The 'forward star' 42 , within which the forward direction is searched, comprises 15 nodes horizontally and 30 nodes vertically. Numerical tests demonstrate further increasing the size of the 'forward star' has minor influence on the calculated rays and travel times. We use the tomographic algorithm described in ref. 43 to invert Pn arrivals. Roughness penalties ${ }^{43}$ are used to smooth the tomographic model. The strengths of first- and second-order smoothing penalties are tested and selected in each iteration to avoid the introduction of artefacts. Considering the vertical velocity variation is dominant for the upper mantle, the horizontal smoothing scale length is set to three times greater than the vertical smoothing scale length to highlight the velocity variation in depth. The initial $\chi^{2}$ before tomography is 18.0. The tomography process is terminated at $\chi^{2}=2$.2. Further reducing $\chi^{2}$ introduces ray streaks and local artefacts, especially in the region just below the Moho. The initial and final travel time residuals are compared in Supplementary Fig. 4. The plot of ray distributions in the mantle shows the maximum sampling depth of rays is $\sim 60 \mathrm{~km}$ below the sea surface (Supplementary Fig. 5).

Influence of the vertical smearing effect on the tomographic result. The tomographic result from the OBS data shows that the LVA at the Romanche TF extends to $60 \mathrm{~km}$ depth below the sea surface (Fig. 3a). 
Because of the potential for the vertical smearing of features during tomographic inversion ${ }^{7}$, we investigate whether the LVA truly extends to $60 \mathrm{~km}$ depth, or it is only a result of vertical smearing. The smearing tests are performed using the initial model, by inserting LVAs with different widths and vertical extents into the mantle beneath the Romanche TF. The maximum depths of the introduced LVAs are 30 $\mathrm{km}, 40 \mathrm{~km}$ and $50 \mathrm{~km}$ and their widths are $30 \mathrm{~km}$ or $50 \mathrm{~km}$. The inserted LVAs have a constant velocity reduction of $8 \%$. We calculate the travel times for these simulated velocity models using the same sourcereceiver configuration as that used in the tomography of the OBS data, and add some random travel time noise to mimic the picking uncertainty. Then we invert these synthetic travel time datasets using a similar 2-D tomography workflow (the same iteration process, smoothing strategy and parameters, initial model and similar final $\chi^{2}$ ) as that used in the tomography of OBS dataset. The tomographic results using the synthetic datasets are shown in Supplementary Fig. S6. Red lines in Supplementary Fig. S6 represent the base of the introduced LVAs, and any velocity reductions below the red lines are created by the downward smearing effect. Supplementary Fig. $\mathrm{S} 6$ demonstrates that the amplitude of velocity reduction caused by the downward smearing effect is less than $0.5 \%$ below $35 \mathrm{~km}$ depth. The downward smearing effect slightly enhances as the width of the introduced LVA increases, but cannot produce a $1 \%$ of velocity reduction at 50-60 km depths. These results demonstrate that a LVA extending to $\sim 60 \mathrm{~km}$ depth at the Romanche TF is required by the OBS data, and hence real, and is not due to the vertical smearing effect of tomography.

\section{Resolution study for the width and velocity reduction of the LVA beneath the Romanche TF. Ray-based}

travel time tomography generally provides a smoothed version of the structure of the subsurface. In order to evaluate the resolution of the tomographic model from the OBS data, we conduct two groups of tests using synthetic seismic datasets. LVAs with different widths and amplitudes of velocity reduction are introduced into the initial model that was used in the tomography of the OBS data, extending from the Moho down to the base of the model. The width of the inserted LVA is constant in depth in each test. Similar to the smearing tests, synthetic travel time datasets are generated using these designed models, and are inverted using a 2-D tomography workflow.

In the first group of tests, we fix the amplitude of velocity reduction within the introduced LVA to $8 \%$ and vary the width of the LVA from $25 \mathrm{~km}$ to $60 \mathrm{~km}$ (Supplementary Fig. 7). We take $8 \%$ of velocity reduction for the inserted LVAs because the maximum velocity reduction within the LVA obtained using the OBS data is $\sim 8 \%$ (Fig. 3a). When the width of the introduced LVA is $25 \mathrm{~km}$, the recovered velocity anomaly at the Moho is $\sim 5 \%$, with no recovery at $60 \mathrm{~km}$ depth (Supplementary Fig. 7a). As the width of the introduced LVA increases, both the percentage and depth of the recovered velocity anomaly increase (Supplementary Figs. 7b-d). When the width of the LVA is $45-50 \mathrm{~km}$, the $8 \%$ velocity reduction is recovered at the Moho, with $\sim 1 \%$ recovered velocity anomaly at 50-60 km depths (Supplementary Figs. 7e,f), similar to the observed LVA derived from the tomography of the OBS data (Fig. 3a). Further increasing the width of the introduced LVA to $60 \mathrm{~km}, \sim 2 \%$ of anomaly at $50 \mathrm{~km}$ depth is recovered (Supplementary Fig. $7 \mathrm{~g}$ ).

In the second group of tests, we take a LVA with a constant width of $50 \mathrm{~km}$ as an example to study the influence of the amplitude of velocity reduction of the LVA on the tomographic result. We vary the 
percentage of velocity reduction within the LVA from $3 \%$ to $9 \%$ (Supplementary Fig. 8). For an introduced LVA with less than $7 \%$ velocity anomaly, $<1 \%$ of velocity anomaly is recovered at $60 \mathrm{~km}$ depth (Supplementary Fig. 8a-e). When the amplitude of velocity reduction of the inserted LVA is $8 \%, \sim 8 \%$ velocity anomaly is recovered at the Moho, with $\sim 1 \%$ recovered velocity anomaly at $60 \mathrm{~km}$ depth (Supplementary Fig. 8f). Further increasing the amplitude of the velocity reduction to $9 \%$, the introduced LVA can be better recovered (Supplementary Fig. 8g).

These tests suggest that the recovered $8 \%$ velocity anomaly just below the Moho at the Romanche TF using the OBS dataset requires a $50 \mathrm{~km}$ wide LVA with at least $8 \%$ velocity decrease at this depth. A larger velocity reduction is required if the width of the LVA is narrower than $50 \mathrm{~km}$, due to the existence of strong tradeoff between the width and the amplitude of velocity reduction in tomography. In all the tests, the majority of the LVAs is not completely recovered, suggesting that the tomography method underestimates the amplitude of the velocity anomaly significantly. The failure of constructing the sharp boundaries of the LVA using OBS data can possibly be attributed to the horizontal smoothing effect of tomographic inversion, which is clearly observed in all the tomographic results from the synthetic datasets (Supplementary Figs. 7,8). From these tests, we are able to get some constraints on the possible width and amplitude of velocity reduction of the real LVA beneath the Romanche TF. Since the amplitude of the introduced velocity anomaly is kept constant for the whole column in each test, the recovered vertical mantle velocity gradient beneath the Romanche TF decreases smoothly with depth (Supplementary Figs. 7h,8h), which is different from the inverted velocity gradient using the OBS data. This implies that the velocity reduction of the real LVA beneath the Romanche TF varies with depth.

Resolution tests on LVA with changing velocity reduction with depth. Resolution tests demonstrates that the tomography method under-estimates the amplitude of the velocity anomaly significantly. To quantify the width and velocity reduction of the real LVA beneath the Romanche TF, we perform a series of tests following the concepts of geophysical inversion. Firstly, we design a base mantle velocity model (model A, Supplementary Fig 9) by removing the LVA from the tomographic velocity model from OBS data (Fig. 3a) and replacing it by interpolating mantle velocity of the normal lithosphere on both sides. We then introduce LVA with different widths and changing velocity reduction with depth into model A beneath the Romanche TF to create a suite of models. The depths of $16 \mathrm{~km}$ and $32 \mathrm{~km}$ below the sea surface, where changes in the vertical velocity gradient are observed, together with the Moho and $60 \mathrm{~km}$ depth are used as controlling nodes. The changing velocity reduction with depth is achieved by varying the amplitude of velocity reduction on the controlling nodes. The amplitude of velocity reduction at any depth between two controlling nodes is linearly interpolated from controlling nodes. In each test, the width of the LVA remains constant in depth, because also varying the width would introduce too many parameters into the inversion, making it too difficult to assess the results. The range of the percentage of velocity reduction on each controlling node is determined as follows:

At the Moho: The tomographic mantle velocity (Fig. 3a) from OBS data shows $~ 8 \%$ velocity decrease just below the Moho at the Romanche TF. OBS26, which is deployed within the Romanche transform valley (Supplementary Fig. 10a), recorded high quality Pg, PmP and Pn arrivals (Supplementary Fig. 10b). The 
positive travel time residuals (, Supplementary Fig. 10c) of OBS26 suggest the mantle velocity just below the Moho should be lower. This means the mantle velocity reduction at the Moho beneath the Romanche TF should be larger than $8 \%$. Resolution tests demonstrate that when the amplitude of velocity reduction within the inserted LVA is $8 \%$, an LVA wider than $45 \mathrm{~km}$ can produce an $8 \%$ velocity anomaly at the Moho after tomography (Supplementary Figs. 7e-g). Based on these observations, we limit the maximum width of the inserted LVA to be $45 \mathrm{~km}$ at Moho, with a minimum percentage of velocity reduction of $8 \%$.

The crustal velocity just above the Moho is $6.25 \pm 0.3 \mathrm{~km} / \mathrm{s}$ (ref. 8) and Moho reflections are observed on OBS26 (Supplementary Fig. 10b), thus to enable these reflections the mantle velocity just below the Moho must be larger than $6.25 \pm 0.3 \mathrm{~km} / \mathrm{s}$. In our test, we set the maximum amplitude of velocity reduction just below Moho to $14 \%$, which limits the minimum mantle velocity to $6.75 \mathrm{~km} / \mathrm{s}$.

$16 \mathrm{~km}$ depth: To avoid the possible introduction of a negative vertical mantle velocity gradient within the inserted LVA, we constrain the amplitude of velocity reduction at $16 \mathrm{~km}$ depth as between that at the Moho and at $32 \mathrm{~km}$ depth.

$32 \mathrm{~km}$ depth: The velocity reduction of the recovered LVA using the OBS data is $\sim 2 \%$ at $32 \mathrm{~km}$ depth below the sea surface, where the mantle velocity below the Romanche TF is $\sim 7.80 \mathrm{~km} / \mathrm{s}$ and that beneath the ridge segment is $\sim 7.95 \mathrm{~km} / \mathrm{s}$ at this depth (Fig. 3a). The resolution tests show that if the velocity reduction of the inserted LVA is $8 \%$ at this depth, the LVA cannot be narrower than $30 \mathrm{~km}$ in order to obtain a recovered velocity reduction of $2 \%$ (Supplementary Fig. $7 \mathrm{~b}$ ), Furthermore, it should not be wider than $50 \mathrm{~km}$, otherwise the recovered velocity reduction is $\sim 3 \%$ at $32 \mathrm{~km}$ depth (Supplementary Fig. 7f). When the inserted LVA is $50 \mathrm{~km}$ wide, resolution tests show that more than $4 \%$ of velocity reduction within the inserted LVA is required to produce $\sim 2 \%$ of recovered velocity anomaly at $32 \mathrm{~km}$ depth (Supplementary Figs. 8b,c). Based on these observations, we set the width range of the inserted LVA at 32 $\mathrm{km}$ depth to $30-50 \mathrm{~km}$, with the amplitude of velocity reduction varying from $4 \%$ to $8 \%$.

$60 \mathrm{~km}$ depth: We assume the amplitude of velocity reduction at $60 \mathrm{~km}$ depth shares the same range as that at $32 \mathrm{~km}$ depth due to the nearly constant tomographic velocity gradient between $32 \mathrm{~km}$ and $60 \mathrm{~km}$ depth observed in the tomographic result from OBS data (Fig. 3b). The width of the LVAs used in tests is constant in depth, so varies between $30 \mathrm{~km}$ to $50 \mathrm{~km}$.

Based on the above analysis, we create a suite of models (Synthetic models in Supplementary Fig. 11) by inserting LVAs with different widths and changing velocity reductions in depth (Supplementary Table 1) into model A (Supplementary Fig 9). We calculate travel times through these synthetic models using the same source-receiver configuration as that used in the tomography of the OBS data. The picking uncertainties of the OBS data are assigned to these modelled travel time datasets, in order to eliminate the influence of picking errors on the estimated width and velocity of the LVA. These synthetic travel time datasets are inverted starting from the same initial model as that used in the tomography of the OBS dataset. After tomography, all these synthetic travel time datasets produce velocity models (Inverted results in Supplementary Fig. 11) showing similar mantle velocity structure to the tomographic result using the OBS data (Fig. 3a) with a LVA in the mantle beneath the Romanche TF. The difference between 
the LVA obtained using the OBS dataset (Fig. 3a) and those from the synthetic datasets (Inverted results in Supplementary Fig. 11) are measured by L2-norm

$$
J=\left\|m_{\text {coss }}-m_{s y n}\right\|_{2}^{2} \text {. }
$$

To compare the velocity difference between the LVAs, we design two windows to select different regions of the LVAs. Window 1 covers the region between horizontal distance $410 \mathrm{~km}$ to $430 \mathrm{~km}$ and depth from the Moho to $25 \mathrm{~km}$ depth below the sea surface, and is used to constrain the width and mantle velocity at the Moho and at $16 \mathrm{~km}$ depth. Window 2 with horizontal distance from $400 \mathrm{~km}$ to $440 \mathrm{~km}$ and depth from $25 \mathrm{~km}$ to $60 \mathrm{~km}$ is used to constrain the width and velocity of the LVA at $32 \mathrm{~km}$ and $60 \mathrm{~km}$ depths.

Supplementary Table 1 shows the velocity misfits measured within different windows. We set a misfit threshold to select the synthetic models whose tomographic results have a good fit to the OBS data inverted result. By taking the average of the width and velocity of the introduced LVA in the selected models, we estimate the width and amplitude of velocity reduction of the real LVA below the Romanche TF (Supplementary Table 2). The uncertainties of the width and amplitude of velocity reduction are characterized by their first standard deviations. Different misfit thresholds yield very similar estimates, which validates the stability of our method. In this work, we choose the estimate model parameters obtained using a misfit threshold of 20 (17 models selected for Window 1 and 22 models selected for Window 2 (Supplementary Table 1)) as the final result that is used in the interpretation. Our result (Fig. $3 \mathrm{c}$ ) shows that the width of the real LVA beneath the Romanche TF varies from $34.1 \pm 3.9 \mathrm{~km}$ at the Moho to $35.6 \pm 5.7 \mathrm{~km}$ at $60 \mathrm{~km}$ depth below sea surface, with the mantle velocity within the LVA being $6.89 \pm 0.12 \mathrm{~km} / \mathrm{s}, 7.34 \pm 0.08 \mathrm{~km} / \mathrm{s}, 7.52 \pm 0.10 \mathrm{~km} / \mathrm{s}$ and $7.75 \pm 0.06 \mathrm{~km} / \mathrm{s}$ at the Moho and at $16 \mathrm{~km}, 32 \mathrm{~km}$ and $60 \mathrm{~km}$ depths, respectively.

Finally, we create a synthetic model (Supplementary Fig. 12a) by inserting a LVA with the estimated velocity and width into Model A (Supplementary Fig 9). We calculate and then invert travel times calculated using this synthetic model, and the tomographic model (Supplementary Fig. 12b) is compared with that from the OBS data (Fig. 3a). The comparisons give a misfit of 4.5 in Window 1 and 3.8 in Window 2 , which demonstrates that the result produced by this inversion is a closer fit to the final OBS data inversion result than the 60 synthetic models listed in Supplementary Table 1.

Temperature and pressure calculation. The temperature of the lithosphere north and south of the Romanche TF is estimated using a plate cooling model ${ }^{22}$, assuming a uniform water thickness of $5 \mathrm{~km}$. The plate thickness ${ }^{18}$ is set to $106 \mathrm{~km}$ and the oceanic mantle potential temperature ${ }^{44}$ is $1410^{\circ} \mathrm{C}$. We assume a $35 \mathrm{~km}$-wide shear zone with vertical boundaries beneath the Romanche TF. The temperature of the lithosphere beneath the Romanche TF is estimated by averaging the temperature of the lithosphere on both sides. The temperature in the center of the shear zone is used as the temperature of the Romanche TF. The estimated temperatures at Moho and at $16 \mathrm{~km}, 32 \mathrm{~km}$ and $60 \mathrm{~km}$ depths are $250^{\circ} \mathrm{C}$, $450^{\circ} \mathrm{C}, 920^{\circ} \mathrm{C}$ and $1260^{\circ} \mathrm{C}$, respectively (Supplementary Fig. 13). 
The pressures at different depths below the Romanche TF are estimated using a model comprising a 5 $\mathrm{km}$-thick water column and a $6 \mathrm{~km}$-thick crust overlying the mantle. The densities of the crust and mantle are set to $2800 \mathrm{~kg} / \mathrm{m}^{3}$ and $3150 \mathrm{~kg} / \mathrm{m}^{3}$, respectively. The density of mantle used in the pressure estimation is smaller than the undepleted peridotite ( $3250 \mathrm{~kg} / \mathrm{m}^{3}$ (ref. 23)), due to the presence of hydrous minerals and melt within the mantle that we interpret in this study. The estimated pressures at Moho and at $16 \mathrm{~km}, 32 \mathrm{~km}$ and $60 \mathrm{~km}$ depths are $0.21 \mathrm{GPa}, 0.37 \mathrm{GPa}, 0.86 \mathrm{GPa}$ and $1.73 \mathrm{GPa}$, respectively.

Serpentinization calculation. To estimate the amount of serpentine in the upper mantle beneath the Romanche TF, we assume the melt-impregnated peridotite as the dominant peridotite within the fault zone above $16 \mathrm{~km}$ depth, since melt impregnation is commonly observed in the dredged peridotites along the Romanche $\mathrm{TF}^{45}$, 46 . The melt-impregnated peridotite used in calculation comprises $57.6 \%$ olivine ( $90 \%$ Fo, $10 \% \mathrm{Fa}$ ), $16.5 \%$ orthopyroxene ( $90 \%$ En, $10 \%$ Fs), $11.8 \%$ clinopyroxene (Di), $1.5 \%$ spinel and $11 \%$ plagioclase $(90 \% \mathrm{An}, 10 \% \mathrm{Ab})$, which are obtained by averaging the compositions of the peridotite samples listed in Tartarotti et al. ${ }^{46}$. We assume the anisotropy of the melt-impregnated peridotite is $4 \%$, as a stronger mantle anisotropy is expected within TFs as compared to the average anisotropy $(3.4 \pm 0.3 \%)$ of the normal oceanic lithosphere in the Atlantic Ocean ${ }^{47}$. Assuming that the olivine-toserpentine alteration is the dominant reaction in serpentinization, the velocities in ridge-parallel direction of $0 \%$ and $100 \%$ serpentinized melt-impregnated peridotite at the reference temperature $\left(100^{\circ} \mathrm{C}\right)$ and pressure $(0.01 \mathrm{GPa})$ condition are $7.88 \mathrm{~km} / \mathrm{s}$ and $6.13 \mathrm{~km} / \mathrm{s}$, respectively, estimated using the Matlab toolbox from Abers et al. ${ }^{23}$. The variations of temperature and pressure with depth relative to the reference temperature and pressure cause changes of $-0.04 \mathrm{~km} / \mathrm{s}$ and $-0.12 \mathrm{~km} / \mathrm{s}$ in P-wave velocity at the Moho and at $16 \mathrm{~km}$ depth ${ }^{23}$, respectively. After the temperature-pressure correction, the estimated $6.89 \pm 0.12 \mathrm{~km} / \mathrm{s}$ and $7.34 \pm 0.08 \mathrm{~km} / \mathrm{s}$ mantle velocities at the Moho and $16 \mathrm{~km}$ depth (Fig. $3 \mathrm{c}$ ) correspond to $55 \pm 7 \%$ and $25 \pm 5 \%$ of serpentinization (Supplementary Fig. 14), respectively.

The pressure used in the calculation has a negligible influence on the estimated mantle serpentinization, as increasing pressure by $0.1 \mathrm{GPa}\left(\sim 3.2 \mathrm{~km}\right.$ in depth) leads to $\sim 0.01 \mathrm{~km} / \mathrm{s}$ velocity change ${ }^{23}$. When increasing temperature by $100^{\circ} \mathrm{C}$, the seismic velocity of the melt-impregnated peridotite decreases by $\sim 0.04 \mathrm{~km} / \mathrm{s}$ (ref. 23), and the estimated mantle serpentinization will decrease by $\sim 3 \%$. Using stronger anisotropy for the melt-impregnated peridotites will yield a lower estimate of serpentinization. For example, using $5 \%$ anisotropy for the melt-impregnated peridotite in these calculations, the estimated mantle serpentinization is $52 \pm 7 \%$ and $22 \pm 5 \%$ at the Moho and $16 \mathrm{~km}$ depth, respectively.

Hydration and mylonitization calculation. Chlorite and amphibole are the main hydrous minerals at medium temperature $\left(T \leq 750^{\circ} \mathrm{C}\right)$ and only amphibole crystalizes at higher temperatures ${ }^{19,20}$. To estimate the fractions of hydrous phases that cause the mantle velocity decrease in the mylonitic zone, we assume that the hydrated lithosphere is composed of melt-impregnated peridotites and hydrous minerals, as melt-impregnation is suggested to occur shallower than $\sim 32 \mathrm{~km}$ depth below the sea level ${ }^{14}$. 
We use the same composition for the melt-impregnated peridotite as for the serpentinization calculations to estimate the degree of hydration at medium temperature. For higher temperature $\left(T>750^{\circ} \mathrm{C}\right)$, we use the melt-impregnated peridotite with a composition of $58.8 \%$ olivine ( $90 \% \mathrm{Fo}, 10 \% \mathrm{Fa}$ ), $7.45 \%$ orthopyroxene ( $90 \%$ En, 10\% Fs), $23.85 \%$ clinopyroxene (Di), 1.6\% spinel and $8.3 \%$ plagioclase ( $90 \% \mathrm{An}, 10 \% \mathrm{Ab}$ ). The composition of the high-temperature melt-impregnated peridotite is obtained by averaging the compositions of three high-temperature melt-impregnated peridotite samples from Tartarotti et al. ${ }^{46}$.

We assume the anisotropy of the melt-impregnated peridotite is $4 \%$. P- and S-wave anisotropies of chlorite used in the calculations are $27 \%$ and $56 \%$, respectively ${ }^{48}$. P- and S-wave anisotropies of amphibole are $9.6 \%$ and $7.0 \%$, respectively, which are obtained from the analysis of four mylonites dredged from TFs in the Southwest Indian Ocean ${ }^{19}$. Natural sampled medium-temperature $\left(T \leq 750^{\circ} \mathrm{C}\right)$ mylonites comprise on average of $16 \%$ chlorites and $24 \%$ amphiboles ${ }^{19}$. In our calculations, we assume the relative amount of chlorite and amphibole formed at medium temperature is 2:3.

The elastic parameters of the melt-impregnated peridotite, chlorite and amphibole are obtained and extrapolated to high temperature-pressure conditions using the Matlab toolbox from Abers et al. ${ }^{23}$. Mantle velocity variation for increasing amounts of hydrous phases is estimated using an effective medium analysis ${ }^{26}$.

Supplementary Fig. 15a shows the variation in mantle velocity as a function of the total amount of chlorite and amphibole at $16 \mathrm{~km}$ depth, where the predicted temperature is $450^{\circ} \mathrm{C}$ (Supplementary Fig. 13). A total of $28 \pm 6 \%$ hydrous minerals are required to account for the low mantle velocity at $16 \mathrm{~km}$ depth, which means $70 \pm 15 \%$ of the lithospheric mantle is mylonitic at this depth (Supplementary Fig. 15b).

At $32 \mathrm{~km}$ depth where the predicted temperature is $920^{\circ} \mathrm{C}$ (Supplementary Fig. 13 ), $10 \pm 7 \%$ amphibole within the mantle is required to account for the estimated mantle velocity of $7.52 \pm 0.10 \mathrm{~km} / \mathrm{s}$ (Supplementary Fig. 15c). Natural sampled high-temperature peridotite mylonites comprise 12.5\% amphibole on average ${ }^{19}$, and this means that at least $24 \%$ of the lithospheric mantle at $32 \mathrm{~km}$ depth is mylonitic (Supplementary Fig. 15d).

As with the serpentinization calculations, the pressure used in these calculations has a negligible influence on the estimated mantle hydration. At temperatures higher than $450^{\circ} \mathrm{C}$, an increase of $100^{\circ} \mathrm{C}$ decreases the seismic velocity of the melt-impregnated peridotite by $\sim 0.05 \mathrm{~km} / \mathrm{s}$ (ref. 23), and so decreases the estimated mantle hydration at $16 \mathrm{~km}$ and $32 \mathrm{~km}$ depths by $4 \%$. Using stronger anisotropy for the melt-impregnated peridotite will give a lower estimate of mantle hydration, where an increase of $1 \%$ in mantle anisotropy causes a decrease of $~ 3 \%$ in estimated mantle hydration.

Excess temperature computation. To examine whether the velocity reduction between $32 \mathrm{~km}$ to $60 \mathrm{~km}$ depth is caused by the thermal diffusion from a hotter to a colder lithosphere across the TF, we calculate the excess temperature required for explaining the velocity reduction at this depth range. 
We take the temperature and the average velocity of the 40 Myr-old lithosphere north of the Romanche TF as benchmarks, and assume the velocity reduction below $32 \mathrm{~km}$ depth is only caused by the temperature variation. We first estimate the derivative of mantle velocity with respect to temperature and its variation at depth ${ }^{49}$. Our calculation shows that the derivative of mantle velocity with respect to temperature below $32 \mathrm{~km}$ depth has minor variations with temperature and depth: less than $1 \%$ for a $100^{\circ} \mathrm{C}$ increase in temperature or $20 \mathrm{~km}$ change in depth. In this case, we assume the mantle velocity derivative with respect to temperature is constant $\left(-0.525 \mathrm{~m} / \mathrm{s} /{ }^{\circ} \mathrm{C}\right)$ below $32 \mathrm{~km}$ depth. The velocity decrease within the fault zone (Supplementary Fig. 16a) is obtained by computing the difference between the best estimated mantle velocity of the LVA (Figs. 3c) and the average mantle velocity below the 40 Myr-old lithosphere (Figs. 3a). The excess temperature (Supplementary Fig. 16b) at a specific depth is estimated by dividing the average velocity reduction to the mantle velocity derivative with respect to temperature. To explain the velocity reduction below $32 \mathrm{~km}$ depth, the temperature of the lithosphere would need to increase by $\sim 680$ $820^{\circ} \mathrm{C}$, making the temperature of the lithosphere higher than $1260^{\circ} \mathrm{C}$, which defines the upper boundary of the thermally controlled LAB in the Mid-Atlantic Ocean ${ }^{15}$. However, this high temperature is not possible, because the lithospheric mantle would melt under this temperature, suggesting that the velocity anomaly below $32 \mathrm{~km}$ depth cannot be explained by excess temperature, and some other mechanism is required to explain the observation.

Melt fraction estimation. Melt fractions between $32 \mathrm{~km}$ and $60 \mathrm{~km}$ depths are estimated using the effective medium theory ${ }^{26}$. Melt ellipsoids with aspect ratio ranging from 1 to 100 are considered in the estimation. We assume the P-wave velocity in the ridge-parallel direction of the background matrix of mantle is $8.2 \mathrm{~km} / \mathrm{s}$. The melt inclusions are assumed to be isolated from each other, and this assumption holds for a low concentration of melt. The density and elastic parameters of melt are taken from Clark et al. ${ }^{50}$. In this work, we assume the aspect ratio of melt inclusions is 50 , based on the assumption that melt inclusions are in the form of thin film. Therefore, the estimated melt fractions at $32 \mathrm{~km}$ depth and $60 \mathrm{~km}$ depth are $1.75 \pm 0.30 \%$ and $1.11 \pm 0.16 \%$ (Supplementary Fig. 17), respectively.

Using a larger P-wave velocity for the surrounding rocks will yield a higher estimate of the melt fraction, where increasing the P-wave velocity of the surrounding rocks by $0.1 \mathrm{~km} / \mathrm{s}$ increases the estimated melt fraction by $\sim 0.22 \%$. More melt is required to account for the estimated seismic velocity when using a lower aspect ratio of melt inclusion in the calculation (Supplementary Fig. 17).

Water fraction estimation. The water content within the serpentinized and mylonitic zones can be estimated using the amounts of serpentine, chlorite or amphibole and the amount of water these hydrous phases contain. The serpentine, chlorite and amphibole used in calculations contain $12.3 \mathrm{wt} \%, 13.0 \mathrm{wt} \%$ and $2.2 \mathrm{wt} \%$ of water ${ }^{23}$, respectively. At the Moho and $16 \mathrm{~km}$ depth, the water contents estimated using serpentine only are $6.77 \pm 0.86 \mathrm{wt} \%$ and $3.08 \pm 0.62 \mathrm{wt} \%$, respectively. Using chlorites and amphiboles, the estimated water contents at 16 and $32 \mathrm{~km}$ depths are $1.81 \pm 0.39 \mathrm{wt} \%$ and $0.22 \pm 0.15 \mathrm{wt} \%$, respectively. The mismatch of water contents estimated from serpentine and chlorite/amphibole at $16 \mathrm{~km}$ depth may result from the use of inaccurate anisotropies and velocities of peridotites and hydrous minerals, the 
relative amount of chlorite and amphibole formed at medium temperature, or the possible co-existence of these hydrous minerals.

The temperatures $\left(920-1260^{\circ} \mathrm{C}\right.$; Supplementary Fig. 13) defining the water-induced melt zone are lower than the dry solidus of lithospheric mantle ${ }^{27}$, so volatiles such as water or carbon dioxide are needed to trigger hydrous mantle melting 27,28 . Because the influence of carbon dioxide on the solidus of lithospheric mantle is not well constrained, we only consider water as the volatile between $32 \mathrm{~km}$ and 60 $\mathrm{km}$ depth. The water contents required to enable hydrous melting of mantle at different pressuretemperature conditions are estimated using the model described in Katz et al. ${ }^{27}$

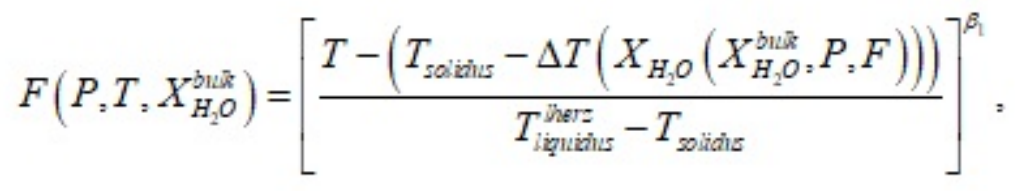

where $T$ and $P$ are the temperature and pressure at depth, $T_{\text {solidis }}$ is the solidus

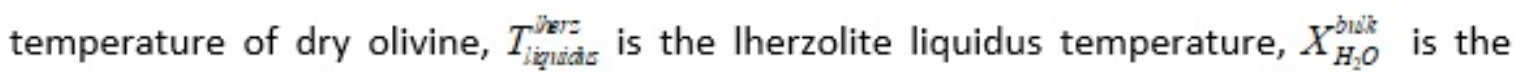
bulk water fraction in wt\% and $X_{\mathrm{H}_{2} \mathrm{O}}$ is the weight fraction of water in melt, $\Delta T\left(X_{\mathrm{H}_{2} \mathrm{O}}\right)$ represents the temperature decrease of the solidus due to the presence of $X_{H_{2}} \mathrm{O}$ water in the melt. $\beta_{1}$ is the power-law exponent.

All the values of these parameters are taken from Katz et al. ${ }^{27}$. Melt fractions of $1.75 \mathrm{wt} \%$ and $1.11 \mathrm{wt} \%$ are used in the estimation of water contents at 32 and $60 \mathrm{~km}$ depths, respectively. The solidus of lithosphere with different bulk contents of water is shown in Supplementary Fig. 18. The minimum temperature to melt a water-saturated mantle at $32 \mathrm{~km}$ depth is $\sim 922^{\circ} \mathrm{C}$ (ref. 27; Supplementary Fig. 18a), which is very close to the predicted temperature of $920^{\circ} \mathrm{C}$ using the plate cooling model ${ }^{22}$ (Supplementary Fig. 13). In this case, we assume that the mantle beneath the Romanche TF starts to melt at $32 \mathrm{~km}$ depth, and the water content required is $0.32 \mathrm{wt} \%$ (Supplementary Fig. 18a). At $60 \mathrm{~km}$ depth where the estimated temperature is $1260^{\circ} \mathrm{C}$ (Supplementary Fig. 13), the required water content is 0.02 wt\% (Supplementary Fig. 18b).

Supplementary Fig. 19 demonstrates that the water content in the mantle below the Romanche TF decreases with depth. For the water-induced melt zone below the transform valley, water content decreasing with depth is consistent with the water content variation in the LAB ${ }^{15}$.

\section{Declarations}

\section{Acknowledgements}


We are grateful to the officers, crew, and scientific technicians of the 2018 ILAB-SPARC cruise for their hard work. The discussions with Daniele Brunelli and Barbara Romanowicz were extremely useful. The research leading to these results has received funding from the European Research Council under the European Union's Seventh Framework Programme (FP7/2007-2013)/ ERC Advance Grant agreement no. 339442_TransAtlanticlLAB. This is an Intitut de Physique du Globe de Paris contribution number XXXX.

\section{Author contributions}

Z.W. processed the OBS data and wrote the paper. S.C.S. developed the project, led the data acquisition and supervised the data processing, interpretation and writing. C.P. participated in the calculations. E.P.M.G. and M.M. participated in the data acquisition, data processing and calculations. All authors discussed results and contributed to the manuscript.

\section{Competing interests}

The authors declare that they have no competing interests.

\section{Data availability.}

The high-resolution bathymetry data collected during the ILAB-SPARC expedition and presented in this paper is available online (https://doi.pangaea.de/10.1594/PANGAEA.922331) under the condition of acknowledging Marjanović et al., 2020. The travel time picks will be available along with publication of the paper and the raw OBS seismic data used in this study on request from the authors.

\section{References}

1. Wilson JT. A New Class of Faults and their Bearing on Continental Drift. Nature 1965, 207: 343-347.

2. Hensen C, Duarte JC, Vannucchi P, Mazzini A, Lever MA, Terrinha P, et al. Marine Transform Faults and Fracture Zones: A Joint Perspective Integrating Seismicity, Fluid Flow and Life. Frontiers in Earth Science 2019, 7(39).

3. Gutscher M-A. What Caused the Great Lisbon Earthquake? Science 2004, 305(5688): 1247-1248.

4. Bird P. An updated digital model of plate boundaries. Geochemistry, Geophysics, Geosystems 2003, 4(3).

5. Bird P, Kagan YY, Jackson DD. Plate tectonics and earthquake potential of spreading ridges and oceanic transform faults. Plate Boundary Zones, Geodynamics Series 2002, 30: 203-218.

6. Cooper GF, Macpherson CG, Blundy JD, Maunder B, Allen RW, Goes S, et al. Variable water input controls evolution of the Lesser Antilles volcanic arc. Nature 2020, 582(7813): 525-529.

7. Roland E, Lizarralde D, McGuire JJ, Collins JA. Seismic velocity constraints on the material properties that control earthquake behavior at the Quebrada-Discovery-Gofar transform faults, East Pacific Rise. J Geophys Res 2012, 117(B11). 
8. Gregory E, Marjanović M, Wang Z, Singh S. Structure and composition of large-offset Atlantic transform faults: an extreme example at the Romanche transform from wide-angle refraction data. EGU General Assembly 2020 2020: EGU2020-10151.

9. Abercrombie RE, Ekström G. Earthquake slip on oceanic transform faults. Nature 2001, 410(6824): 74-77.

10. DeMets C, Gordon RG, Argus DF. Geologically current plate motions. Geophys J Int 2010, 181(1): 180.

11. Norris RJ, Toy VG. Continental transforms: A view from the Alpine Fault. Journal of Structural Geology 2014, 64: 3-31.

12. Bassetto M, Alkmim FF, Szatmari P, Mohriak WU. The Oceanic Segment of the Southern Brazilian Margin: Morpho-Structural Domains and Their Tectonic Significance. Atlantic Rifts and Continental Margins, 2000, pp 235-259.

13. Müller RD, Sdrolias M, Gaina C, Roest WR. Age, spreading rates, and spreading asymmetry of the world's ocean crust. Geochemistry, Geophysics, Geosystems 2008, 9(4).

14. Bonatti E, Brunelli D, Fabretti P, Ligi M, Portaro RA, Seyler M. Steady-state creation of crust-free lithosphere at cold spots in mid-ocean ridges. Geology 2001, 29(11): 979-982.

15. Mehouachi F, Singh SC. Water-rich sublithospheric melt channel in the equatorial Atlantic Ocean. Nature Geoscience 2018, 11(1): 65-69.

16. de Melo GWS, Parnell-Turner R, Dziak RP, Smith DK, Maia M, do Nascimento AF, et al. Uppermost Mantle Velocity beneath the Mid-Atlantic Ridge and Transform Faults in the Equatorial Atlantic Ocean. Bulletin of the Seismological Society of America 2020.

17. Marjanović M, Singh SC, Gregory EPM, Grevemeyer I, Growe K, Wang Z, et al. Seismic Crustal Structure and Morpho-tectonic Features Associated with the Chain Fracture Zone and their Role in the Evolution of the Equatorial Atlantic Region. J Geophys Res 2020.

18. McKenzie D, Jackson J, Priestley K. Thermal structure of oceanic and continental lithosphere. Earth and Planetary Science Letters 2005, 233(3): 337-349.

19. Prigent $\mathrm{C}$, Warren JM, Kohli AH, Teyssier C. Fracture-mediated deep seawater flow and mantle hydration on oceanic transform faults. Earth and Planetary Science Letters 2020, 532: 115988.

20. Kohli AH, Warren JM. Evidence for a Deep Hydrologic Cycle on Oceanic Transform Faults. J Geophys Res 2020, 125(2): e2019JB017751.

21. Guillot S, Schwartz S, Reynard B, Agard P, Prigent C. Tectonic significance of serpentinites. Tectonophysics 2015, 646: 1-19.

22. Stein CA, Stein S. A model for the global variation in oceanic depth and heat flow with lithospheric age. Nature 1992, 359(6391): 123-129.

23. Abers GA, Hacker BR. A MATLAB toolbox and Excel workbook for calculating the densities, seismic wave speeds, and major element composition of minerals and rocks at pressure and temperature. Geochemistry, Geophysics, Geosystems 2016, 17(2): 616-624. 
24. Yu Z, Singh SC, Gregory EPM, Maia M, Wang Z, Brunelli D. Semi-brittle seismic deformation in hightemperature mantle mylonite shear zone along the Romanche transform fault. Science Advances 2021: Accepted.

25. Lizarralde D, Gaherty JB, Collins JA, Hirth G, Kim SD. Spreading-rate dependence of melt extraction at mid-ocean ridges from mantle seismic refraction data. Nature 2004, 432: 744.

26. Taylor MAJ, Singh SC. Composition and microstructure of magma bodies from effective medium theory. Geophys J Int 2002, 149(1): 15-21.

27. Katz RF, Spiegelman $\mathrm{M}$, Langmuir $\mathrm{CH}$. A new parameterization of hydrous mantle melting. Geochemistry, Geophysics, Geosystems 2003, 4(9).

28. Keller T, Katz RF. The Role of Volatiles in Reactive Melt Transport in the Asthenosphere. Journal of Petrology 2016, 57(6): 1073-1108.

29. Bonatti E, Ligi M, Gasperini L, Peyve A, Raznitsin Y, Chen YJ. Transform migration and vertical tectonics at the Romanche fracture zone, equatorial Atlantic. J Geophys Res 1994, 99(B11): 2177921802.

30. Wang S, Constable S, Rychert CA, Harmon N. A Lithosphere-Asthenosphere Boundary and Partial Melt Estimated Using Marine Magnetotelluric Data at the Central Middle Atlantic Ridge. Geochemistry, Geophysics, Geosystems 2020, 21(9): e2020GC009177.

31. Hékinian R, Bideau D, Hébert R, Niu Y. Magmatism in the Garrett transform fault (East Pacific Rise near 13²7'S). J Geophys Res 1995, 100(B6): 10163-10185.

32. Roland E, Behn MD, Hirth G. Thermal-mechanical behavior of oceanic transform faults: Implications for the spatial distribution of seismicity. Geochemistry, Geophysics, Geosystems 2010, 11(7).

33. Ligi M, Bonatti E, Cipriani A, Ottolini L. Water-rich basalts at mid-ocean-ridge cold spots. Nature 2005, 434(7029): 66-69.

34. Menard HW, Atwater T. Origin of Fracture Zone Topography. Nature 1969, 222(5198): 1037-1040.

35. Müller RD, Landgrebe TCW. The link between great earthquakes and the subduction of oceanic fracture zones. Solid Earth 2012, 3(2): 447-465.

36. Wei S, Helmberger D, Avouac J-P. Modeling the 2012 Wharton basin earthquakes off-Sumatra: Complete lithospheric failure. J Geophys Res 2013, 118(7): 3592-3609.

37. Coudurier-Curveur A, Karakaş Ç, Singh S, Tapponnier P, Carton H, Hananto N. Is There a Nascent Plate Boundary in the Northern Indian Ocean? Geophys Res Lett 2020, 47(7): e2020GL087362.

38. ten Brink US, Al-Zoubi AS, Flores CH, Rotstein Y, Qabbani I, Harder SH, et al. Seismic imaging of deep low-velocity zone beneath the Dead Sea basin and transform fault: Implications for strain localization and crustal rigidity. Geophys Res Lett 2006, 33(24).

39. Fuis GS, Kohler MD, Scherwath M, Brink UT, Avendonk HJAV, Murphy JM. A Comparison Between the Transpressional Plate Boundaries of South Island, New Zealand, and Southern California, USA: The Alpine and San Andreas Fault Systems. A Continental Plate Boundary: Tectonics at South Island, New Zealand, 2007, pp 307-327. 
40. Fichtner A, Saygin E, Taymaz T, Cupillard P, Capdeville Y, Trampert J. The deep structure of the North Anatolian Fault Zone. Earth and Planetary Science Letters 2013, 373: 109-117.

41. Bécel A, Laigle M, de Voogd B, Hirn A, Taymaz T, Galvé A, et al. Moho, crustal architecture and deep deformation under the North Marmara Trough, from the SEISMARMARA Leg 1 offshore-onshore reflection-refraction survey. Tectonophysics 2009, 467(1): 1-21.

42. Moser TJ. Shortest path calculation of seismic rays. Geophysics 1991, 56(1): 59-67.

43. Van Avendonk HJA, Shillington DJ, Holbrook WS, Hornbach MJ. Inferring crustal structure in the Aleutian island arc from a sparse wide-angle seismic data set. Geochemistry, Geophysics, Geosystems 2004, 5(8).

44. Sarafian E, Gaetani GA, Hauri EH, Sarafian AR. Experimental constraints on the damp peridotite solidus and oceanic mantle potential temperature. Science 2017, 355(6328): 942-945.

45. Seyler M, Bonatti E. Regional-scale melt-rock interaction in Iherzolitic mantle in the Romanche Fracture Zone (Atlantic Ocean). Earth and Planetary Science Letters 1997, 146(1): 273-287.

46. Tartarotti P, Susini S, Nimis P, Ottolini L. Melt migration in the upper mantle along the Romanche Fracture Zone (Equatorial Atlantic). Lithos 2002, 63(3): 125-149.

47. Gaherty JB, Lizarralde D, Collins JA, Hirth G, Kim S. Mantle deformation during slow seafloor spreading constrained by observations of seismic anisotropy in the western Atlantic. Earth and Planetary Science Letters 2004, 228(3): 255-265.

48. Mookherjee M, Mainprice D. Unusually large shear wave anisotropy for chlorite in subduction zone settings. Geophys Res Lett 2014, 41(5): 1506-1513.

49. Goes $S$, Govers R, Vacher P. Shallow mantle temperatures under Europe from $P$ and $S$ wave tomography. J Geophys Res 2000, 105(B5): 11153-11169.

50. Clark AN, Lesher CE, Jacobsen SD, Wang Y. Anomalous density and elastic properties of basalt at high pressure: Reevaluating of the effect of melt fraction on seismic velocity in the Earth's crust and upper mantle. J Geophys Res 2016, 121(6): 4232-4248.

\section{Figures}




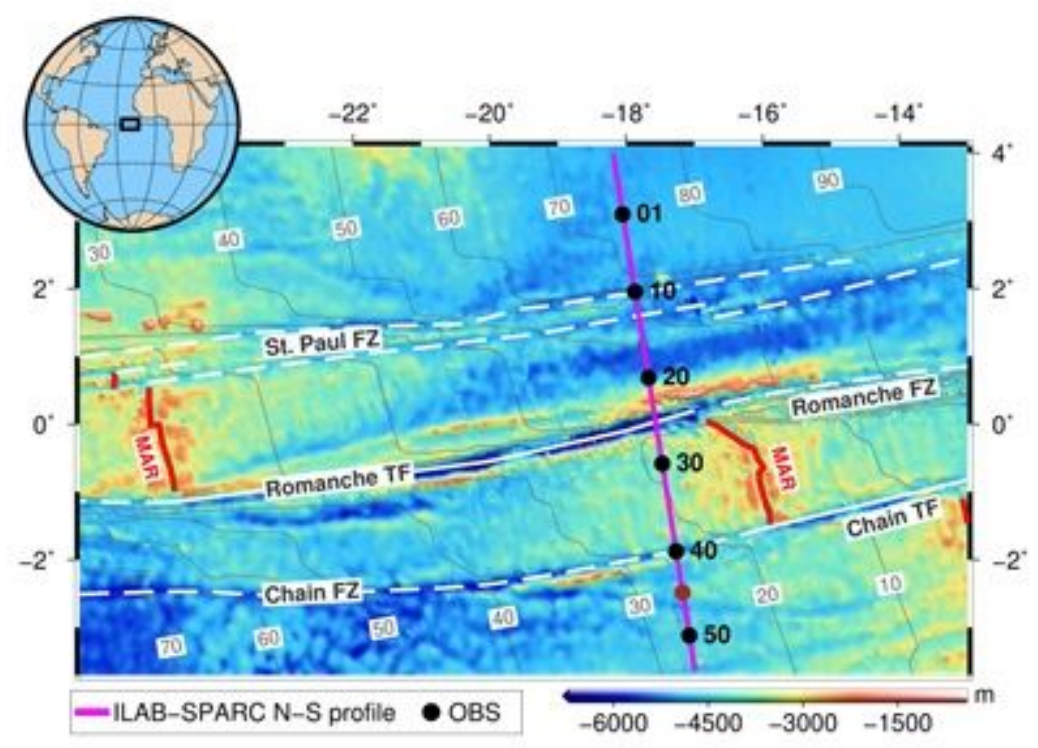

\section{Figure 1}

Study area. Bathymetry map showing the major TFs (white solid lines) and FZs (white dashed lines) in the equatorial Atlantic Ocean. The bathymetry map is plotted by superimposing the high-resolution bathymetry data collected during the ILAB-SPARC experiment on a global low-resolution grid. The seismic refraction profile is shown as a magenta line, with every tenth OBS locations marked by black dots. The brown dot shows the location of OBS45, which records mantle refraction arrivals up to $700 \mathrm{~km}$ offset shown in Fig. 2. Plate age13 contours are plotted and labelled every $10 \mathrm{Myr}$. The black rectangle in the globe inset shows the location of the study area. MAR: Mid-Atlantic ridge; TF: transform fault; FZ: fracture zone.

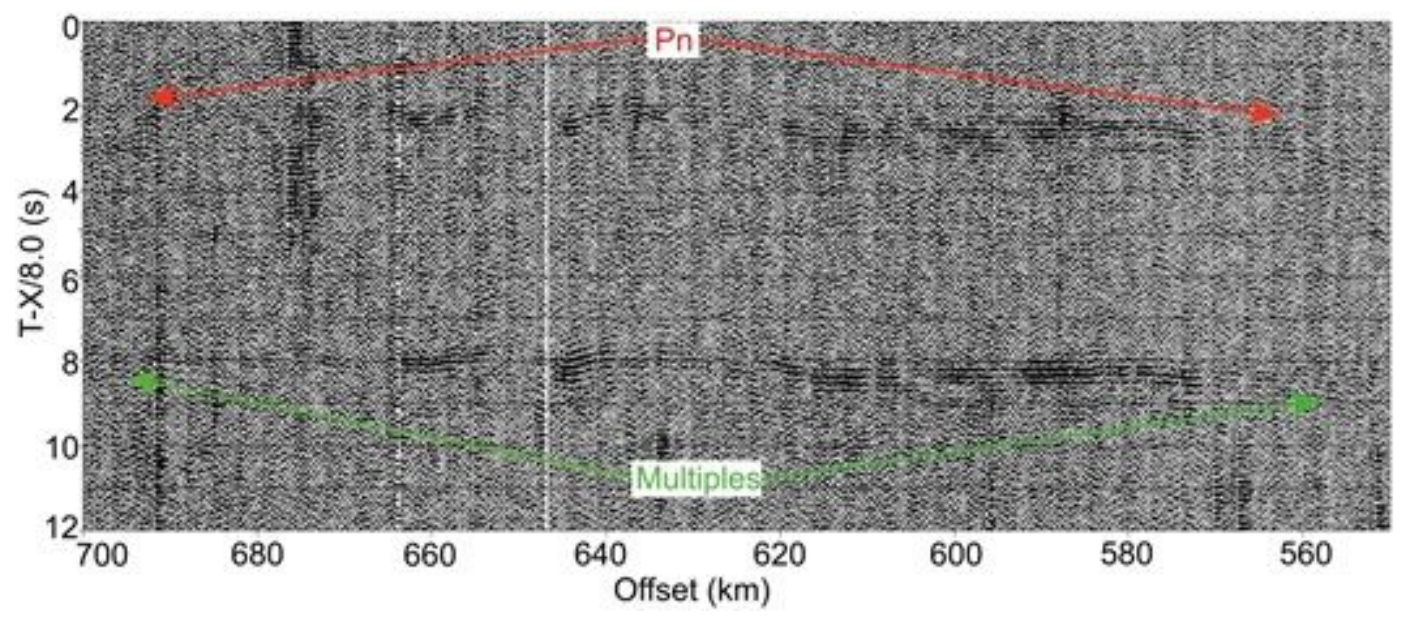

\section{Figure 2}

Ultra-long offset seismic data. Pressure data recorded by OBS45 is plotted at the reduced travel time using a reduction velocity of $8.0 \mathrm{~km} / \mathrm{s}$. The location of OBS45 is shown by the brown dot in Fig. 1 . The effect of bathymetry variation is removed by subtracting the propagating time in water, assuming a water velocity of $1.5 \mathrm{~km} / \mathrm{s}$. The mantle first arrivals $(\mathrm{Pn})$ and first-order multiples are labelled. 


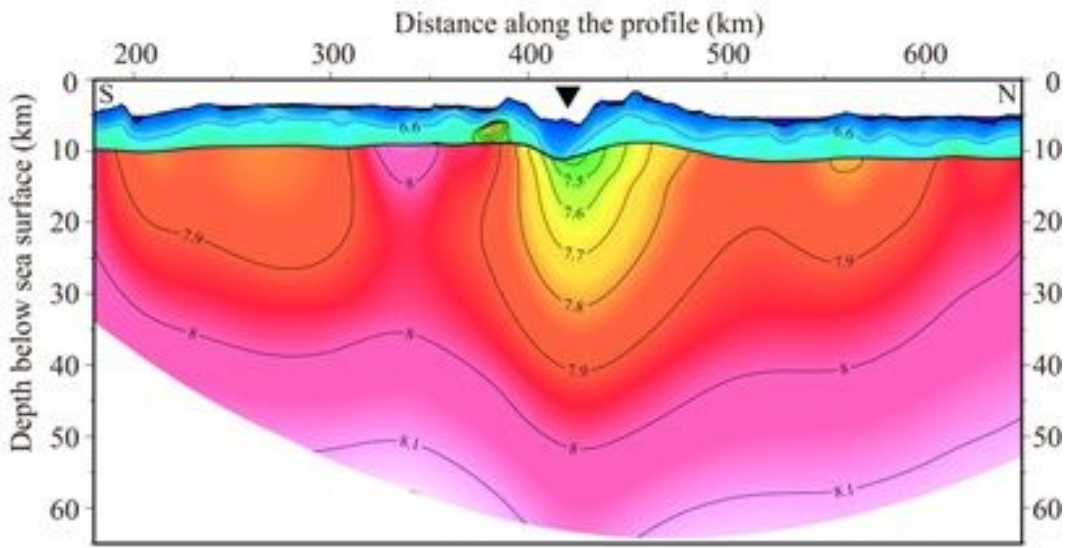

b

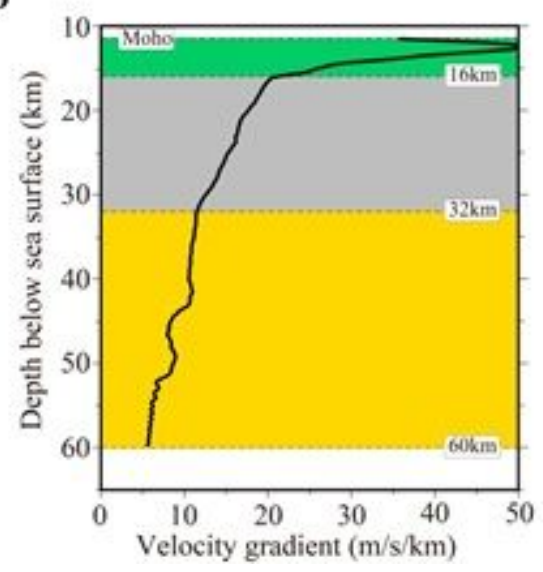

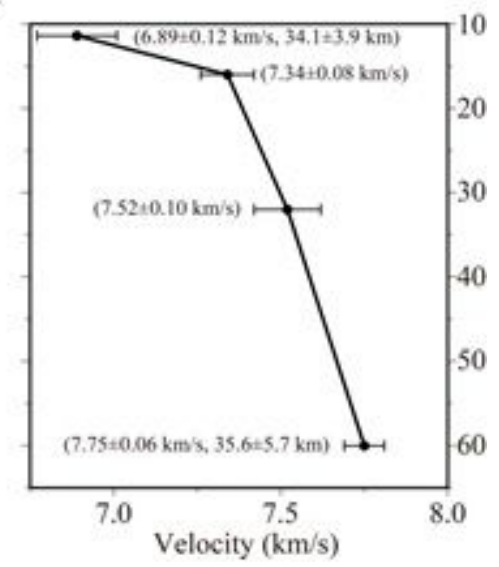

Figure 3

Seismic tomographically estimated and simulated velocity structures of the mantle beneath the Romanche TF. a, Crustal and mantle velocity structures obtained from 2-D ray-based travel time tomography using the OBS data. The inverted black triangle indicates the location of the transform valley. b, Average vertical mantle velocity gradient at the center of the low velocity anomaly (LVA), at 417-425 km horizontal distance in Fig. 3a. Colored sections indicate the three different zones of lithospheric mantle beneath the Romanche TF, defined by the change in the velocity gradient (see the text). c, The best simulated width and mantle velocity of the LVA beneath the Romanche TF and their uncertainties (see Methods). 


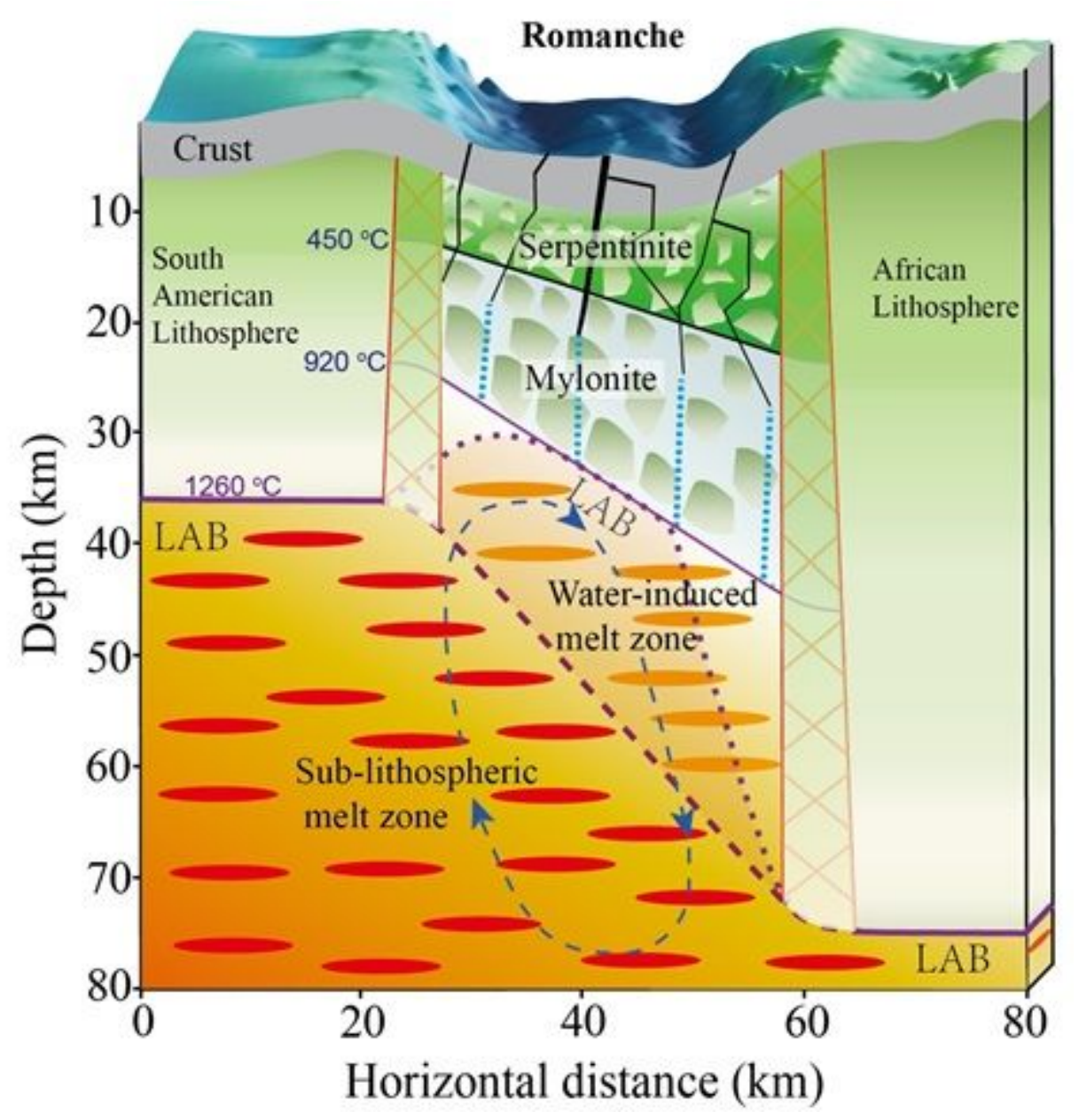

Figure 4

Nature of the lithosphere across the Romanche TF. Schematic diagram showing the lithospheric structure across the Romanche TF. The $450 \mathrm{oC}$ and $920 \mathrm{oC}$ isotherms across the Romanche TF are indicated by thin black and purple lines, respectively, interpreted as the bases of the serpentinized and mylonitic/hydrous peridotite zones. The blue dashed lines indicate the downward water percolation promoted by semi-brittle deformations in the mylonite zone. The regions marked by red crosses represent the transition from the fault zone to the normal oceanic lithosphere. Its structure is not well constrained. The thick purple lines mark the $1260 \mathrm{oC}$ isotherm for the normal oceanic lithosphere, interpreted as the top of the LAB15. The purple dashed line indicates the $1260 \mathrm{oC}$ isotherm below the TF, which is obtained by averaging the temperatures of lithosphere on both sides, separating the water-induced melt zone above with the sublithospheric melt channel below. The interpreted upper boundary of the LAB beneath the Romanche TF (thin purple line) gradually deepens across the TF from the South American plate to the African plate. The purple dotted line is probably closer to the structure of the top of the LAB beneath the Romanche TF. The dark blue dashed arrow demonstrates possible small-scale convection cell.

\section{Supplementary Files}

This is a list of supplementary files associated with this preprint. Click to download. 
- WangetalSupplementaryNatureGeoscience.docx 